\title{
Link Quality Prediction for 802.11 MANETs in Urban Microcells
}

\author{
Gregor Gaertner, Eamonn 0. Nuallain \\ School of Computer Science and Statistics, Trinity College, Dublin, Ireland \\ Email: eamonn.onuallain@scss.tcd.ie
}

How to cite this paper: Gaertner, G. and Nuallain, E.O. (2016) Link Quality Prediction for 802.11 MANETs in Urban Microcells. Journal of Computer and Communications, 4, 61-77. http://dx.doi.org/10.4236/jcc.2016.413005

Received: July 25, 2016

Accepted: October 23, 2016

Published: October 26, 2016

Copyright $\odot 2016$ by authors and Scientific Research Publishing Inc. This work is licensed under the Creative Commons Attribution International License (CC BY 4.0).

http://creativecommons.org/licenses/by/4.0/

\begin{abstract}
In this paper we derive the optimal link quality predictor (LQPR) whose parameters are estimated from signal power and node speed samples. We propose a fast estimator for these parameters whose computational complexity is three orders lower than that of the optimal estimator with only a slight loss in accuracy thus enabling realtime execution. We show that using the most recent local mean of the signal as a predictor of future signal strength is also a very close approximation to the optimal predictor. This is the central result of this paper. It obviates the need for complex and/or computationally intensive link quality predictors for 802.11 in urban microcells and has the advantage of not requiring node speed information. The LQPRs are evaluated against the lower error bound. We show that the LQPR based on the most recent local mean of the signal predicts the packet reception probability for pedestrians in urban microcells on average with a mean absolute error of $13.47 \%, 16.54 \%$, $18.21 \%$ and $19.38 \%$ for $1 \mathrm{~s}, 2 \mathrm{~s}, 3 \mathrm{~s}$ and $4 \mathrm{~s}$ into the future respectively. This LQP accuracy resembles closely the lower error bound with, for example, a difference of only $2.47 \%$ at $2 \mathrm{~s}$ into the future.
\end{abstract}

\section{Keywords}

Mobile Communications, Propagation, Quality Assurance

\section{Introduction}

Link quality prediction (LQP) is a vital tool for the effective operation of MANETS [1] (pp. 275-286) for which applications range from support for disaster recovery to mobile gaming [2]-[4]. The purpose of LQP is to provide information about the channel so that the best communication route or link can be chosen. Furthermore link degradation can be anticipated by using an LQP for the purpose of switching to another link to maintain channel capacity. 
Thus LQP is the basis for preemptive action, [5], and is therefore a key technology in the successful operation of MANETs. Link quality prediction (LQP) has been shown to be somewhat effective in masking topology changes in wireless mobile ad-hoc networks (MANETS). There is a large body of literature on the topic with methods ranging from the deterministic to the stochastic and to the highly novel. Motivated by the need for real-time execution, LQP algorithms are typically based either explicitly or implicitly (in the case of "location-based" algorithms) path-loss propagation models that are very limited representations of real signal conditions and so bear consequent limitations on predictive accuracy. [6]-[10] are some of the better known examples of these type algorithms. However the statistics of signal fading are typically not considered and as we show in this paper these have a strong bearing on predictive accuracy.

We seek to address these weaknesses in this paper. We argue that large-scale fading, over path-loss and small-scale fading, is the primary determinant of successful shortterm (i.e. within a few seconds) link quality prediction at pedestrian speeds. It is within such a timeframe that the preemptive action referred to above can take place. We derive novel Link Quality Prediction Algorithms (LQPRs) that are based on a link model that includes path loss, large-scale and small-scale fading effects.

We begin with the optimal but unrealizable (in the sense that it cannot be deployed for real-time predictions) predictor, termed the "THEO" predictor which we use as a baseline with which to compare our subsequently derived realizable predictors. The first of these requires a runtime estimation of its parameters, for which we obtain the optimal estimator. However, since it is known that no computationally inexpensive recursive solution exists for this class of estimation problem we introduce a suboptimal but fast estimator that enables efficient runtime estimation of the required parameters. We show that the accuracy of this fast estimator is only slightly reduced compared to that of the optimal estimator while being computationally much more efficient thus allowing for its deployment in real-time prediction. This predictor, termed the distance exponential shadowing (DES) predictor, uses both signal-power and node-speed measurements. Where node speed measurements are unavailable we introduce the simple Last Local Mean (LLM) predictor which relies solely on signal-power measurements. The results given by this predictor are very close to those given by the optimal predictor and so obviate the need for complex/computationally intensive predictors. We assess the accuracy of the DES and LLM predictors against the THEO baseline by simulations in an enhanced NS simulator [11].

This is done over a comprehensive range of scenarios with respect to various factors which we expect may influence LQP accuracy i.e. mobility model, packet length and the influence or otherwise of having only bidirectional communication links. The statistical accuracy of our evaluation is demonstrated by means of the confidence intervals. We observe that the results, in terms of predictive accuracy, given by the LLM predictor are very close to those given by the THEO predictor.

\section{Link Quality Predictors}

In this section we derive three LQPRs that accurately predict the Packet Reception 
Probability (PRP) of future transmitted packets in microcellular urban environments. We make the following assumptions:

- Location information is unavailable. GPS measurements can be unreliable in dense urban environments-particularly so for mobile users.

- Node speed is available. Node speed can be measured accurately for pedestrians with an inexpensive accelerometer chip.

- The relative speed of the two nodes for which an LQP is being performed is assumed to be constant within the interval of acquiring signal strength measurements and the time over which link quality is predicted. Most LQP algorithms for mobile nodes either implicitly or explicitly make this assumption [6] [8]-[10] [12] [13].

- The LQPR should only rely on information that is available to the 802.11 MAC layer since lower-layer information from the 802.11 physical layer is typically cardspecific and thus unavailable to higher layers. While the 802.11 cards that were available to us discard received corrupt packets and their associated signal power at firmware level, we assume that the payload with bit errors and the signal power of corrupt packets is available at MAC level. Such assumption does not violate the 802.11 specification [14] and the decision to dispose of this information at firmware level is made arbitrarily by the manufacturer. For example, this information could be discarded at driver level from where it could be extracted for further use in LQP.

a) Definition of an Optimal Predictor:

Let $X_{t}$ be a single sample of a stochastic process. All samples which are realizations of this stochastic process up to a time $\mathrm{T}$ are given by the vector $\mathrm{X}_{T}=\left(\mathrm{X}_{t=1} \cdots \mathrm{X}_{t=T}\right)^{\mathrm{T}}$.

Given $X_{T}$ then the optimal predictor for $X_{T+\tau}$ is [15] (p. 33):

$$
\tilde{\mathrm{X}}_{T+\tau}=\mathrm{E}\left[\mathrm{X}_{T+\tau} \mid \mathrm{X}_{T}\right]
$$

Such a predictor is optimal in the sense that it has the minimum mean square error of all predictors.

b) Radio Link Model:

The received signal power (in decibels) may be modeled as:

$$
R_{T}=B_{T}+S_{T}+H_{T}
$$

where $B$ is the deterministic path loss, $S$, the large-scale fading process, is a correlated normally distributed zero mean random variable with variance $\sigma_{S}$, and $H_{(\mathrm{dB})}=10 \log _{10} h_{\left(\mathrm{v} \cdot \mathrm{m}^{-1}\right)}^{2}$, the power of the small-scale signal envelope $h_{\left(\mathrm{v} \cdot \mathrm{m}^{-1}\right)}$, where $h_{\left(\mathrm{v} \cdot \mathrm{m}^{-1}\right)}$ is a Ricean distributed random variable.

The autocovariance function of $S$ is given by (Goldsmith, p. 51):

$$
A(\delta)=\sigma_{S}^{2} \rho_{D}^{\delta / D}=\sigma_{S}^{2} \rho_{r e f}^{\delta}
$$

where

$$
\rho_{\text {ref }}=\rho_{D}^{1 / D}
$$

Gudmundson [16] determined by empirical measurements that $\rho_{\text {ref }}=0.3$ with $D=10 \mathrm{~m}$ are appropriate values for urban microcells. 
In MANETs, where all nodes are potentially mobile, the additional displacement $\delta_{[t-\tau, t]}$ of mobile nodes $i$ and $j$ in the time interval $[t-\tau, t]$ is approximated by Wang [17]:

$$
\delta_{[t-\tau, t]} \approx\left|o_{t-\tau, i}-o_{t, i}\right|+\left|o_{t-\tau, j}-o_{t, j}\right|
$$

where $|o|$ denotes the length of vector $o$ in Euclidian space.

c) The Optimal Link Quality Predictor:

We now derive the optimal predictor for the signal power of received packets and we subsequently map this prediction to the Packet Reception Probability (PRP).

From Equation (1) the received signal power is predicted optimally, at time $T$ for the time $T+\tau$ by:

$$
\tilde{R}_{T+\tau \mid T}=\mathrm{E}\left[R_{T+\tau} \mid R_{T}\right]
$$

From Equation (2):

$$
\tilde{R}_{T+\tau \mid T}=B_{T+\tau}+\mathrm{E}\left[S_{T+\tau} \mid S_{T}\right]+\mathrm{E}\left[H_{T+\tau} \mid H_{T}\right]
$$

where

$$
\mathrm{E}[H(t)]=-\frac{10}{\ln 10} \gamma
$$

where $\gamma=0.577216 \cdots$ is Euler's Constant [18].

We now individually assess the influence of path-loss, small-scale fading and largescale fading on short-term prediction.

Path Loss:

where $B$ may be assumed to be constant in the interval $[T, T+\tau]$ then:

$$
\tilde{R}_{T+\tau \mid T}=C_{T+\tau}+\mathrm{E}\left[S_{T+\tau} \mid S_{T}\right]
$$

where:

$$
C_{T}=B_{T}+\mathrm{E}[H(t)]
$$

For the purposes of LQP for prediction horizons of a few seconds at pedestrian speeds it may be reasonably assumed that the path loss is constant in the prediction interval for most scenarios. In cases where this becomes a weaker approximation (i.e. where nodes are closely spaced) there is generally no need for LQP.

d) Small-Scale Fading:

Nakabayashi and Kozono [19] investigated the autocorrelation properties of smallscale wideband signal envelope fading based on the wideband signal propagation model of [20]. They showed that the autocorrelation of the small-scale fading signal envelope is independent of the receiver bandwidth and is given for both, narrowband and wideband signals, by [19] [21] (pp. 73, 74):

$$
\rho\left(\Delta_{t}\right)=J_{0}\left(2 \pi f_{d} \Delta_{t}\right)
$$

where $f_{D}$ is the Doppler frequency, $\Delta_{t}$ is the time lag, and $J_{0}$ is the zeroth-order Bessel function of the first kind. This result was derived analytically and also verified by measurements [19]. 
Figure 1 shows the normalized autocorrelation function of the small-scale fading process. The graph shows that the current sample has no predictive information beyond $0.03 \mathrm{~s}$ for the Doppler frequency given for maximum walking speed. This corresponds to results from the literature which state that the received small-scale signal power is uncorrelated after approximately 0.4 wavelengths [21] (p. 74), which corresponds to a distance of approximately $5 \mathrm{~cm}$ for an 802.11 signal. In any case the amplitude of the small-scale signal is generally too small and varies too rapidly to be of use. It is concluded that the small-scale signal has no useable predictive value for 802.11 MANETS.

e) Large-Scale Fading

Finally, we consider the effect of large-scale fading. Figure 2 shows the normalized

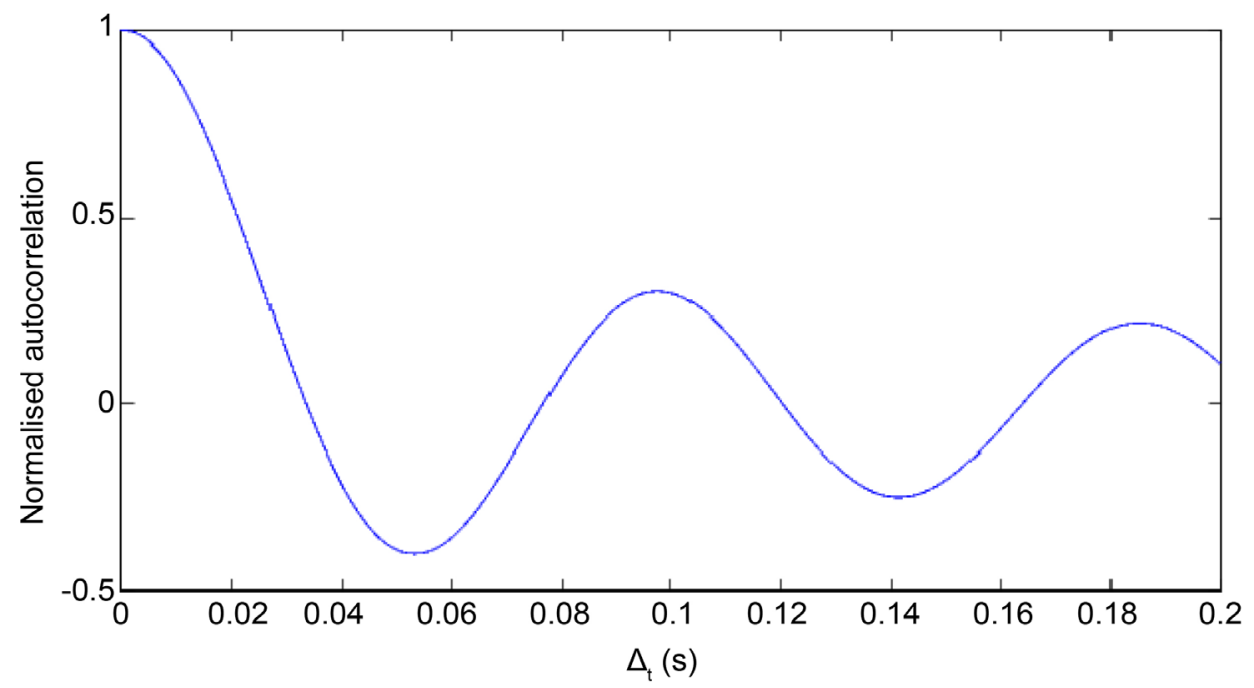

Figure 1. Normalized autocorrelation function for small-scale fading with a Doppler frequency for maximum walking speed $(1.4 \mathrm{~m} / \mathrm{s})$.

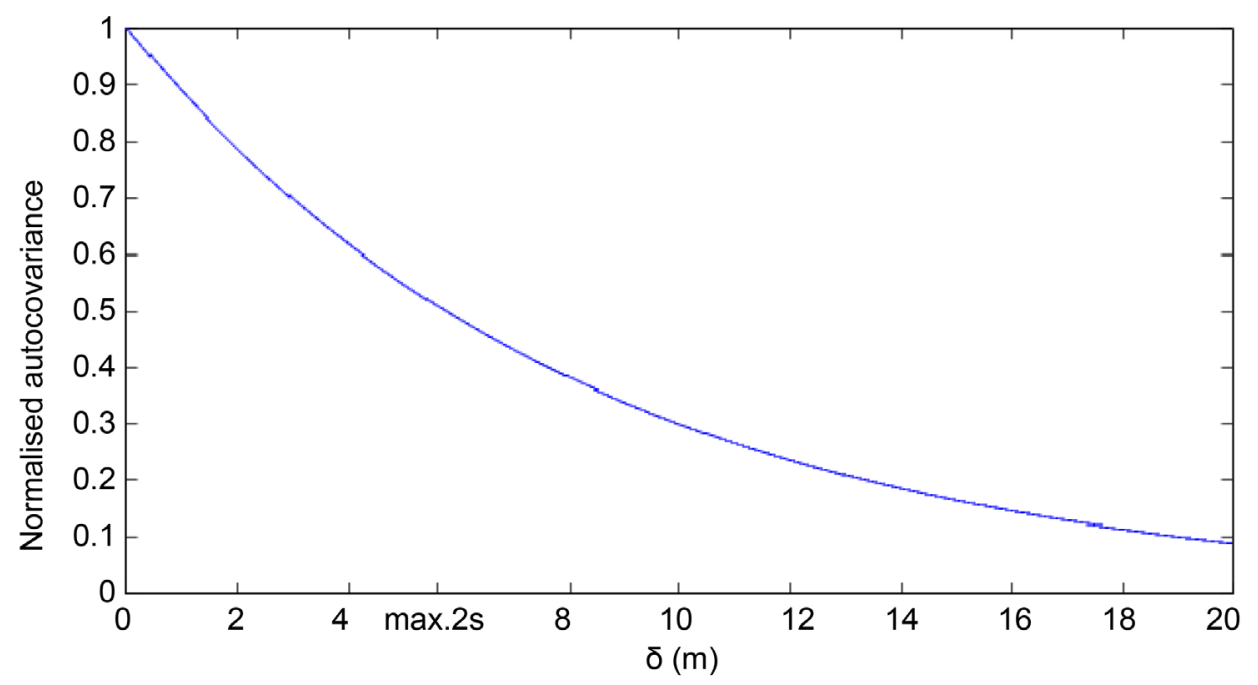

Figure 2. Normalized autocorrelation function for large-scale fading. The maximum distance that two nodes can move apart (at a maximum walking speed of $1.4 \mathrm{~m} / \mathrm{s}$ ) in two seconds is marked on the $\mathrm{X}$-axis. 
autocorrelation function of the large-scale fading signal $\rho_{D}^{\delta / D}$ obtained by Gudmundson [16] who determined by empirical measurements that $\rho_{D}=0.3 \quad(D=10 \mathrm{~m})$ is appropriate for urban microcells. It is clear that there is a high correlation between large-scale fading signal power values for a prediction horizon of up to approximately 2 sec. Given that the path-loss and small-scale fading signal are of little use for 802.11 MANETS in most scenarios we conclude that LQP accuracy for this application is generally dominated by the effects of large-scale fading.

f) Optimal Parameter Estimator:

The optimal predictor of (10) requires that the quantities $C_{T+\tau}$ and $S_{T+\tau}$ are estimated from the sequence of signal-power measurements that are available at the time a prediction is made.

$S$ may be modeled as a first order AR process [22] (p. 100) giving:

$$
\mathrm{E}\left[S_{T+\tau} \mid S_{T}\right] \approx \rho_{r e f}^{\tilde{\delta}_{T+\tau \mid T}} S_{T}
$$

Hence:

$$
\tilde{R}_{T+\tau \mid T} \approx C_{T}+\rho_{r e f}^{\tilde{\delta}_{T+\tau \mid T}} S_{T}
$$

where $\tilde{\delta}_{T+\tau \mid T}$, estimated from the node velocity, is the additional displacement at a prediction horizon $\tau$.

The appropriate averaging interval and sampling rate for an 802.11 signal was shown in [23] to be 5 wavelengths with samples taken at less than 0.5 wavelength intervals. With a maximum walking speed of $1.4 \mathrm{~m} / \mathrm{s}$, this gives a sampling interval of $0.04 \mathrm{~s}$ with the number of samples $N_{H}=11$. The signal power can either be averaged in the logarithmic (decibel) or in the linear (watt) domain with similar accuracy, albeit with slightly better accuracy in the logarithmic domain [24].

Hence:

$$
\tilde{R}_{T}=\frac{1}{N_{H}} \sum_{i=0}^{N_{H}-1} R_{T-i}
$$

Such that:

$$
\tilde{R}_{T} \approx C_{T}+S_{T}
$$

to a best approximation [25].

An estimator is optimal if it attains the Cramer-Rao Lower Bound; thus it has minimum variance, is unbiased and it is impossible to find an unbiased estimator with a lower variance [26] (p. 27). $S$ may be viewed as a normally distributed colored stochastic perturbation about $C$. For this class of model an optimal estimator exists [26] (pp. 83, 94-97):

Let $\mathrm{P}$ denote the sequence of received signal-power samples as a vector of size $N_{C} \times 1$ :

$$
\mathrm{P}=\left[\begin{array}{lllll}
\tilde{R}_{T-N_{C}+1} & \tilde{R}_{T-N_{C}+2} & \cdots & \tilde{R}_{T-1} & \tilde{R}_{T}
\end{array}\right]^{\mathrm{T}}
$$

The optimum estimate for $C_{T}$ is given by [26] (pp. 94, 95): 


$$
\left.\tilde{C}_{T}=\left[\begin{array}{llll}
1 & 1 & \cdots & 1
\end{array}\right] \mathrm{A}(\delta)^{-1}\left[\begin{array}{c}
1 \\
1 \\
\vdots \\
1
\end{array}\right]\right]^{-1} \cdot\left[\begin{array}{llll}
1 & 1 & \cdots & 1
\end{array}\right] \mathrm{A}(\delta)^{-1} \cdot \mathrm{P}
$$

where $\mathrm{A}(\delta)$ is the autocovariance matrix of the large-scale signal power [27] (pp. 370-371):

$$
\mathrm{A}(\delta)=\left[\begin{array}{ccccc}
\sigma_{S}^{2} & \sigma_{S}^{2} \rho_{r e f}^{\left.\delta_{\left[T-N_{C}+1, T-N_{C}+2\right]}\right]} & \cdots & \sigma_{S}^{2} \rho_{r e f}^{\delta_{\left[T-N_{C}+1, T-1\right]}} & \sigma_{S}^{2} \rho_{r e f}^{\delta_{\left[T-N_{C}+1, T\right]}} \\
\sigma_{S}^{2} \rho_{r e f}^{\delta_{\left[T-N_{C}+2, T-N_{C}+1\right]}} & \sigma_{S}^{2} & \cdots & \sigma_{S}^{2} \rho_{r e f}^{\delta_{\left.r-N_{C}+2, T-N_{C}+1\right]}} & \sigma_{S}^{2} \rho_{r e f}^{\delta_{\left[T-N_{C}+2, T-N_{C}+1\right]}} \\
\vdots & \vdots & \ddots & \vdots & \vdots \\
\sigma_{S}^{2} \rho_{r e f}^{\delta_{\left[T-1, T-N_{C}+1\right]}} & \sigma_{S}^{2} \rho_{r e f}^{\delta_{\left[T-1, T-N_{C}+2\right]}} & \cdots & \sigma_{S}^{2} & \sigma_{S}^{2} \rho_{r e f}^{\delta_{[T-1, T]}} \\
\sigma_{S}^{2} \rho_{r e f}^{\delta_{\left[T, T-N_{C}+1\right]}} & \sigma_{S}^{2} \rho_{r e f}^{\delta_{\left[T, T-N_{C}+2\right]}} & \cdots & \sigma_{S}^{2} \rho_{r e f}^{\delta_{[T, T-1]}} & \sigma_{S}^{2}
\end{array}\right]
$$

$\mathrm{A}(\delta)$ is symmetric since $\delta_{[t-\tau, t]}=\delta_{[t, t-\tau]}$. The estimator (24) is also termed a "weighted least-squares estimator" where the weights are given by the covariance matrix [28] (Section 15.2). Having obtained $\tilde{C}_{T}, \tilde{S}_{T}$ is estimated via:

$$
\tilde{R}_{T}=\tilde{C}_{T}+\tilde{S}_{T}
$$

Then:

$$
\tilde{R}_{T+\tau \mid T} \approx \tilde{C}_{T}+\rho_{r e f}^{\tilde{\delta}_{T+\tau \mid T}} \tilde{S}_{T}
$$

g) Computational Complexity of the Optimal Estimator:

The problem with the optimal estimator is its computational cost. It follows directly from the Cramer-Rao Lower Bound that the estimation accuracy of any efficient minimum variance unbiased estimator improves with increasing number of samples $N_{C}$ [29] (p. 219). Although the complexity of the autocovariance matrix inversion has been shown to be of order $O\left(N_{C}^{2.38}\right)$, for very large matrices, practical numerically-stable algorithms are of the order $O\left(N_{C}^{3}\right)$ [30]. Since it is known that for the class of general linear systems with colored noise, to which our link quality model belongs, no optimal estimator exists that can be implemented as a computationally inexpensive recursive solution [26] (p. 248). For this reason we have derive a fast but suboptimal estimatortrading estimation accuracy against computational cost. This will allow for the realtime implementation of the algorithm.

h) The Distance Exponential Shadowing (DES) Predictor:

Fast Parameter Estimator:

The high computational cost of the optimal estimator necessitates finding an accurate suboptimal estimator with a low computational requirement. In order to reduce the computational cost we assume the large-scale fading process is uncorrelated. With this assumption we propose an estimator whose computational complexity is a scalar and compare the results given by this with those of the optimal estimator (20) and assess the impact of this assumption.

Assuming that the large-scale fading process is uncorrelated gives: 


$$
\mathrm{A}(\delta)=\mathrm{I}
$$

where $\mathrm{I}$ is the identity matrix. The following expression for $\tilde{C}_{T}$ results:

$$
\tilde{C}_{T}=\frac{1}{N_{C}}\left[\begin{array}{c}
1 \\
1 \\
\vdots \\
1
\end{array}\right] \mathrm{P}=\frac{1}{N_{C}} \sum_{i=0}^{N_{C}-1} \tilde{R}_{T-i}
$$

As pointed out in [31], any origin-moment based estimator can be computed recursively, giving:

$$
\tilde{C}_{T}=\frac{\left(N_{C}-1\right) \tilde{C}_{T-1}+\tilde{R}_{T}}{N_{C}}
$$

This reduces the complexity of the fast estimator to a scalar.

i) Last Local Mean (LLM) Predictor:

The DES predictor requires that node-speed measurements are available in order to obtain $\tilde{\delta}_{T+\tau \mid T}$. Although such measurements can be easily obtained using a fairly inexpensive accelerometer, incorporating such a component into a device might be considered uneconomic. For this reason we suggest a simple predictor that does not rely on node speed measurements. We observed in our real-world evaluation of LQP algorithms [32] that algorithms based only on the signal-power trend performed much more poorly than algorithms that relied on the local average signal power values only. We therefore propose simply using the most recent estimate of the local mean power as the signal-power prediction i.e.

$$
\tilde{R}_{T+\tau \mid T} \approx \tilde{R}_{T}
$$

The PRP is then obtained by mapping of the predicted signal power to the PRP. We term this PRP predictor the last local mean (LLM) predictor.

\section{Augmenting the NS Simulator}

The objective of our evaluation is to determine the accuracy of the LQPRs derived in this paper in as wide a variety of scenarios as possible for pedestrians in urban environments. This we achieve using an extended NS Simulator [11]. In this paper we use two mobility models (the Random Waypoint Mobility Model (RWP) and the Random Waypoint Mobility Model City Section (CITY). We examine the effect of some of the factors that affect predictive accuracy. A brief summary of the extensions we made to the simulator [11] is given here:

a) Augmenting the Path-Loss Model in the NS Simulator:

To model the path loss we employ the widely used one-slope path-loss model given in Equation (25) with $B_{0}=-28 \mathrm{~dB} \cdot \mathrm{m}, d_{0}=1 \mathrm{~m}$ and $k_{1}=[2.7,3.5]$ as appropriate for urban microcellular environments [21] (pp. 45, 46).

$$
B(d)=-10 k_{1} \log _{10} \frac{d}{d_{0}}+B_{0} \text { for } d_{0}<d
$$

b) Incorporating Correlated Large-Scale Fading Modeling into the NS Simulator: 
To simulate large-scale fading Gudmundson's model [16] is used and can be simulated by first generating a white Gaussian noise process with variance $\sigma_{S}^{2}$ and then passing it through a first-order filter with the response $\rho_{D}^{\delta / D}$ [16] [21] (p. 51), [22] (pp. 99, 100) viz:

$$
S_{t+1}(\delta)=\rho_{D}^{\delta / D} S_{t}+\left(1-\rho_{D}^{\delta / D}\right) N_{t}
$$

where $S$ is the large-scale fading signal power, $\delta$ is the distance variable, $D$ is a reference distance and $N$ is a zero-mean normally distributed random variable. The autocovariance function for $S$ is given by [22] (p. 100):

$$
\rho_{S S}(t, t+1)=\mathrm{E}\left[S_{t} S_{t+1}(\delta)\right]=\sigma_{S}^{2} \rho_{D}^{\delta / D}
$$

where $\sigma_{S}^{2}$ is the variance of the large-scale fading process. $\rho_{D}$ is the normalized autocovariance of the large-scale fading process at a reference distance $D$. We implemented large-scale fading in the NS simulator for urban microcells using $\rho_{D}=0.3$, $D=10 \mathrm{~m}$ and $\sigma_{s}=[3.5,4.0]$ as recommended in [33].

c) Incorporating Bit and Packet Error Modeling into the NS Simulator:

Assuming perfect channel equalization, we now define the average BEP $P_{b}$ for a wideband channel with small-scale fading as the mean of the average BERs over all configurations in a microcellular urban environment by

$$
P_{b}\left(\gamma_{b}\right)=\frac{1}{\left|\Delta L_{\max }, a\right|} \sum_{\Delta L_{\max }=0.1}^{55} \sum_{a=-\infty}^{15} P_{b}^{\prime}\left(\gamma_{b}, g_{k}\left(a, \Delta L_{\max }\right)\right)
$$

where $\left|\Delta L_{\max }, a\right|$ is the total number of configurations and $g_{k}\left(a, \Delta L_{\max }\right)$ is a function that maps the parameters $a, \Delta L_{\max }$ to generate the appropriate Ricean K-factor for a wideband signal [23].

The probability that a packet of $\mathrm{q}$ bits length is successfully received is given by [29] (pp. 108, 109):

$$
\Phi\left(\gamma_{b}, q\right)=\left(1-P_{b}\left(\gamma_{b}\right)\right)^{q}
$$

Substituting for $\gamma_{b}$ gives:

$$
\Phi(R, q)=\left(1-P_{b}\left(\frac{R}{N_{0} D}\right)\right)^{q}
$$

where $\Phi(R, q)$ is the PRP for a packet with MAC data of q bits in length, $P_{b}$ is the average BEP of the wideband channel with small-scale fading (18), $R$ is the received signal power in Watts and $D$ is the data-rate. We implement the packet and bit error calculations using (29) and (31) (Figure 3).

\section{Methodology and Results}

We selected two mobility models for the evaluation: the Random Waypoint Mobility Model (RWP) and the Random Waypoint Mobility Model City Section (CITY). We chose the former since it is the most commonly used mobility model in MANET studies and the latter since it is considered to be a more realistic model [34]. The simulation 


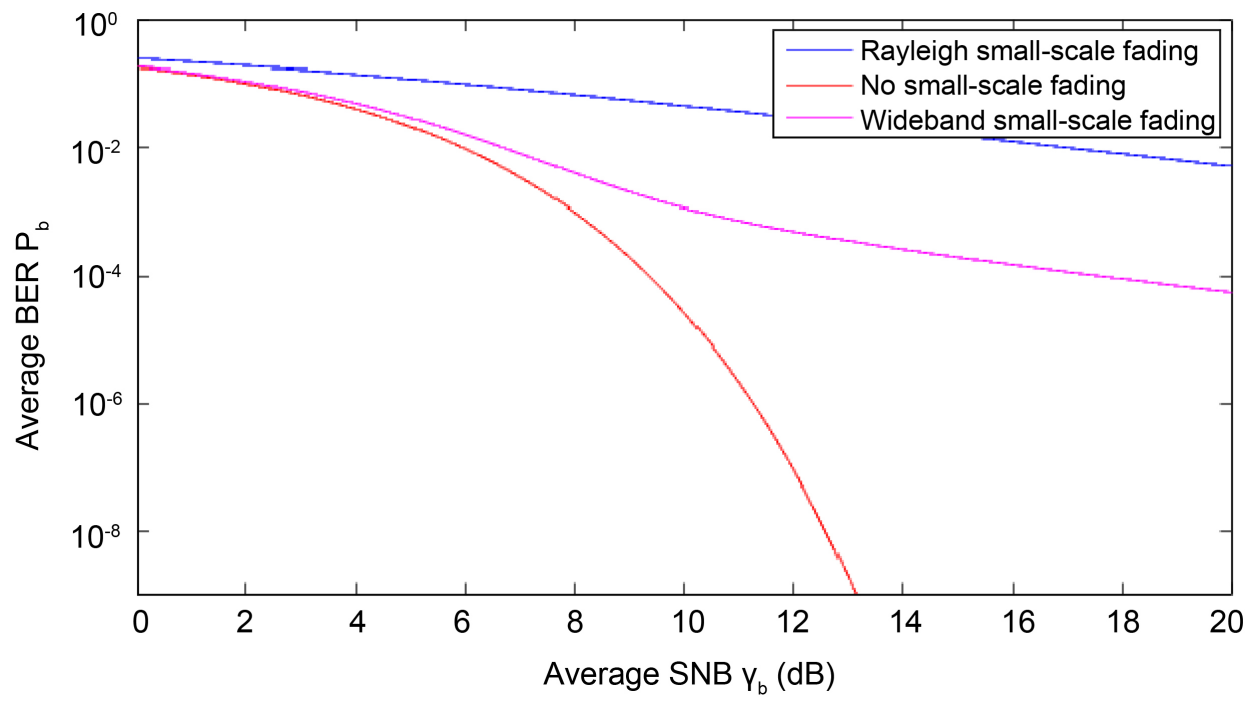

Figure 3. The average BEP for different NLOS channel models vs. the average SNR. The BEP for a channel with wideband small-scale fading lies between the BEP given by a Rayleigh fading channel and a channel without small-scale fading.

area is a 500 msq urban environment, which for the CITY model is a digitally mapped region of the West University, Houston, TX, USA which has been used previously by [35]. For both models a node is assigned a uniformly random chosen speed in the range $[1.2,1.4] \mathrm{m} / \mathrm{s}$ [36] [37]. In the RWP model a pause time is uniform randomly chosen in the range 5-15 sec and the process repeats.

The CITY model operates in a similar way with the following differences. Nodes can move only on pathways which are given by a real-world map. Only the intersections of these pathways can be chosen as destination points thereby limiting the velocity range. Nodes can change frequently change velocity. We set this interval to $15 \mathrm{~s}$. As pointed out by [38] it is of vital importance in MANET studies to perform evaluations only when the evaluation system used has reached steady state. We use a tool given by [34] that provides mobility traces which give steady-state node distributions from the start of the simulation. No prediction and hence no evaluation is performed until enough signal-power samples are available.

The evaluation of our LQPRs is unbiased since each were evaluated in a wide variety of scenarios. To achieve statistical soundness, we use long simulation runs of $3600 \mathrm{~s}$ duration, typically with 5 nodes which is the maximum number of nodes that can be simulated simultaneously using the NS simulator. Each experiment is repeated using 4 independent trials. This effort resulted in approximately 100 million received packets, for which 50 predictions were made and evaluated in the range $[0.08,4.0] \mathrm{s}$.

We evaluate the accuracy of all LQPRs over all permutations for both mobility models using packet lengths of 100, 500, 1000 and 1500 bytes.

Our primary evaluation criterion is the Mean Absolute Error (MAE). We define the absolute error in the prediction as being the difference between the recorded and the predicted PRP at time $T+\tau$ for a packet of MAC payload length $q$ bits in the link 
$(i, j):$

$$
\varepsilon_{\Phi_{T+\tau \mid T}}(i, j, q)=\left|\tilde{\Phi}_{T+\tau \mid T}(i, j, q)-\tilde{\Phi}_{T+\tau \mid}(i, j, q)\right|
$$

The Mean Absolute Error (MAE) of the actual and the predicted PRP for packets of length $\mathrm{q}$ is then:

$$
\bar{\varepsilon}_{\Phi_{\tau}}(q)=\frac{1}{N_{\text {Rounds }} N_{\text {Nodes }}^{2}} \sum_{n=1}^{N_{\text {Rounds }}} \sum_{j=1}^{N_{\text {Nodes }}} \sum_{i=1}^{N_{\text {Nodes }}} \varepsilon_{\Phi_{\tau}}(i, j, n, q)
$$

The evaluation was performed using Matlab, executed on various machines.

We first illustrate how only a small amount of accuracy has been traded in order to achieve the reduction in computational complexity by means of a Monte Carlo simulation. Two nodes are located at points from which they can either move $40 \mathrm{~m}$ towards or away from each other at maximum walking speed. The simulation is repeated 200 times and two scenarios are investigated where the initial displacement of the nodes from one another is $50 \mathrm{~m}$ and $150 \mathrm{~m}$. Figure 4 shows the variation of the Mean Absolute Error (MAE) of the signal-power predictor with prediction horizon. Firstly, it can be observed that the combination of optimal predictor and fast estimator fares only slightly worse in both scenarios than where the combination of optimal predictor and optimal estimator was used. We note that the prediction accuracy at $50 \mathrm{~m}$ starting distance is lower than at $150 \mathrm{~m}$-the approximation of the path loss being constant over the prediction horizon being less accurate where the initial displacement of the nodes from one another is smaller.

a) PRP Accuracy:

The main evaluation criterion for LQP is the predictive accuracy of the PRP since this directly impacts communication quality. This prediction accuracy is given by the MAE of the actual (observed in simulations) and the predicted PRP. As expected the order in decreasing accuracy is the THEO, DES and LLM predictor.

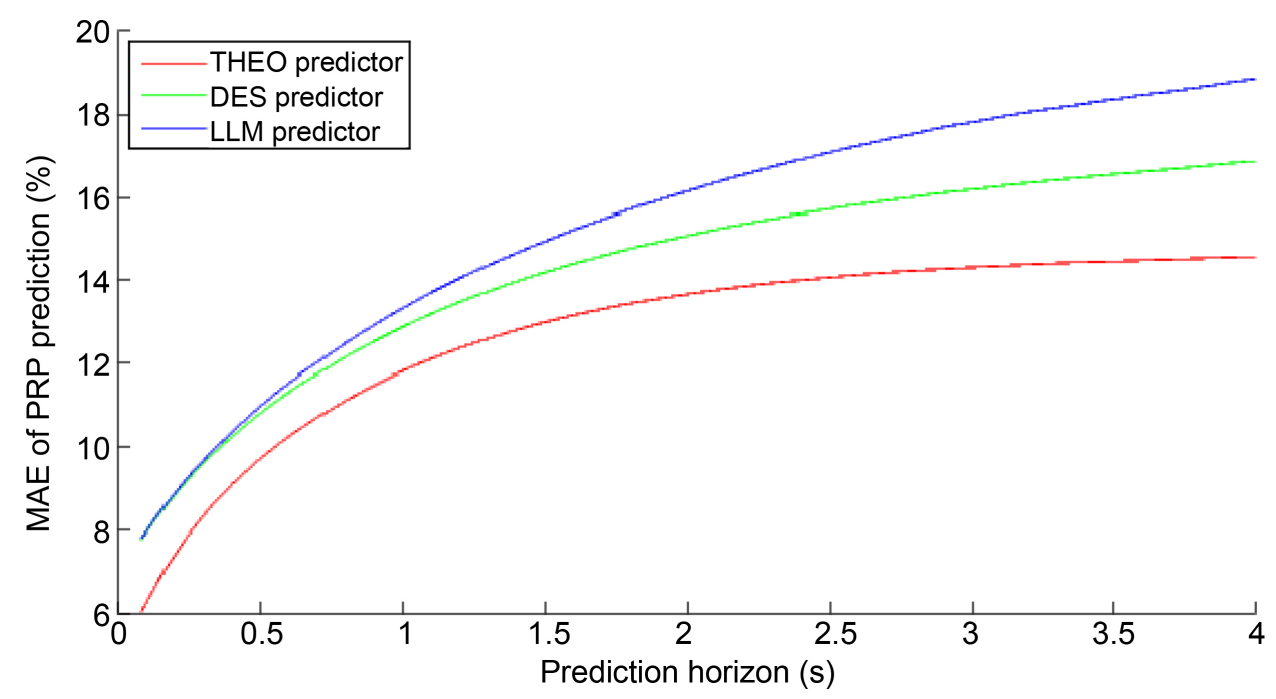

Figure 4. The MAE of PRP prediction versus prediction horizon for the RWP model with 500byte long packets. 
The order of PRP accuracy among the THEO, DES and LLM predictors remains invariant over all evaluated combinations of mobility model and packet length. As expected the LLM predictor gives similar results to the DES for short prediction horizons $(<1 \mathrm{sec})$. The DES predictor gives very satisfactory predictive accuracy (as compared with the THEO baseline). It is evident that little accuracy has been traded for the substantial saving in computational cost thus allowing for the real-time implementation of this algorithm. As is evident from Figure 5 and Figure 6, the RWP mobility model gives slightly lower MAE values than the CITY model. This is also to be expected since the variance in node mobility is greater for the latter. It is also apparent that the MAE decreases with increased packet length.

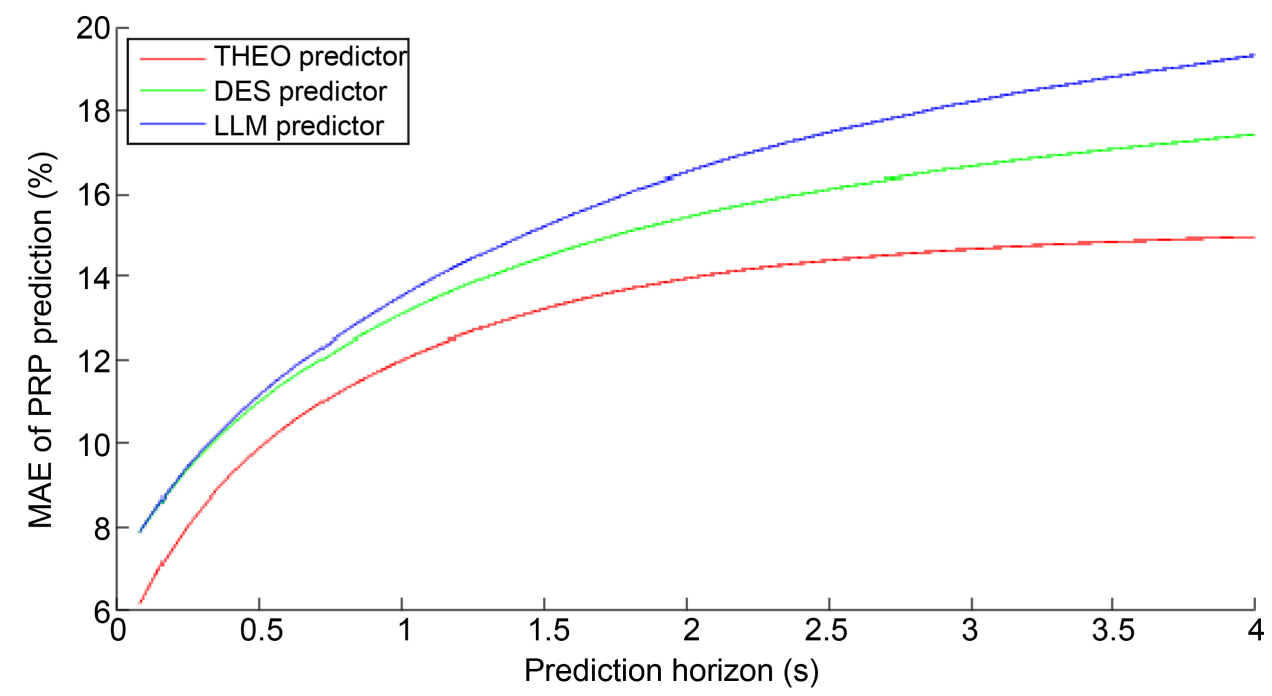

Figure 5. The MAE of PRP prediction versus prediction horizon for the CITY model with 1000byte long packets.

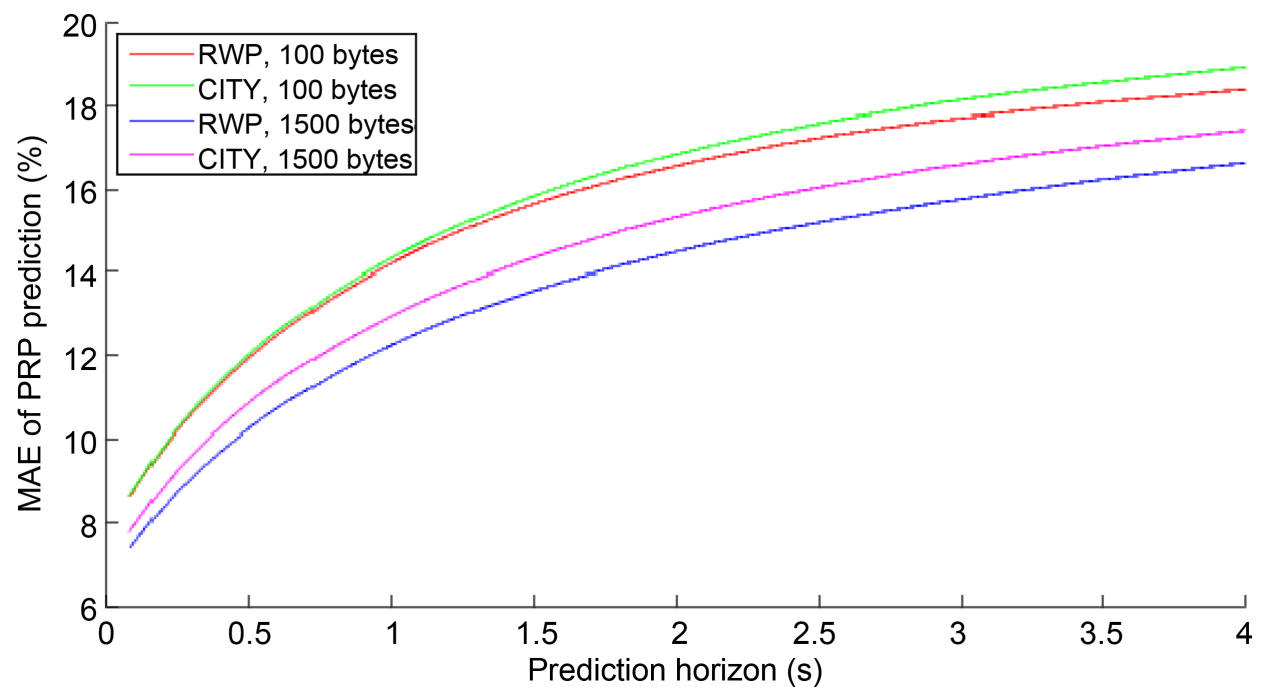

Figure 6. The DES PRP prediction accuracy versus prediction horizon for the CITY and RWP models. 
b) Confidence Intervals:

A credible evaluation requires that its statistical accuracy is shown, which has typically been omitted in most MANET studies [38]. Here, we use confidence intervals to support our evaluation. Figure 7 shows the 95\% confidence interval for scenario SC2-the RWP model with 500-byte long packets, which has been used frequently in the evaluation section. We observe in this and other simulations that the $95 \%$ confidence intervals are narrow, are almost independent of the prediction horizon, the packet length and mobility model. The $95 \%$ confidence intervals are typically within the bounds of $\pm 0.6 \%-0.7 \%$ probability.

\section{Discussion and Conclusion}

In this paper we derived the optimal estimator for link quality prediction based on our link model. The complexity of this estimator (THEO) is $O\left(N^{3}\right)$. This high computational cost precludes the use of this optimal estimator in real-time applications. We derived a sub-optimal but realizable link quality predictor (DES) whose computational complexity is a scalar and observed that our assumptions resulted in little trade-off in accuracy for 802.11 MANETS at pedestrian speeds. This predictor requires both signalpower and node-speed samples from the recent past.

For situations in which no node-speed measurements are available we propose the simple last local mean (LLM) predictor. This is shown to give results very close to that of the optimal predictor in the circumstances described in this paper. We evaluated all LQPRs in a major simulation study in the extended NS simulator. The evaluation baseline is the theoretical (THEO) predictor which gives a lower error bound on LQP that cannot be achieved by any realizable predictor for the given link model. Over a wide range of scenarios we demonstrated that the mean absolute error (MAE) of the packet reception probability (PRP) prediction for the DES predictor is in the worst case within

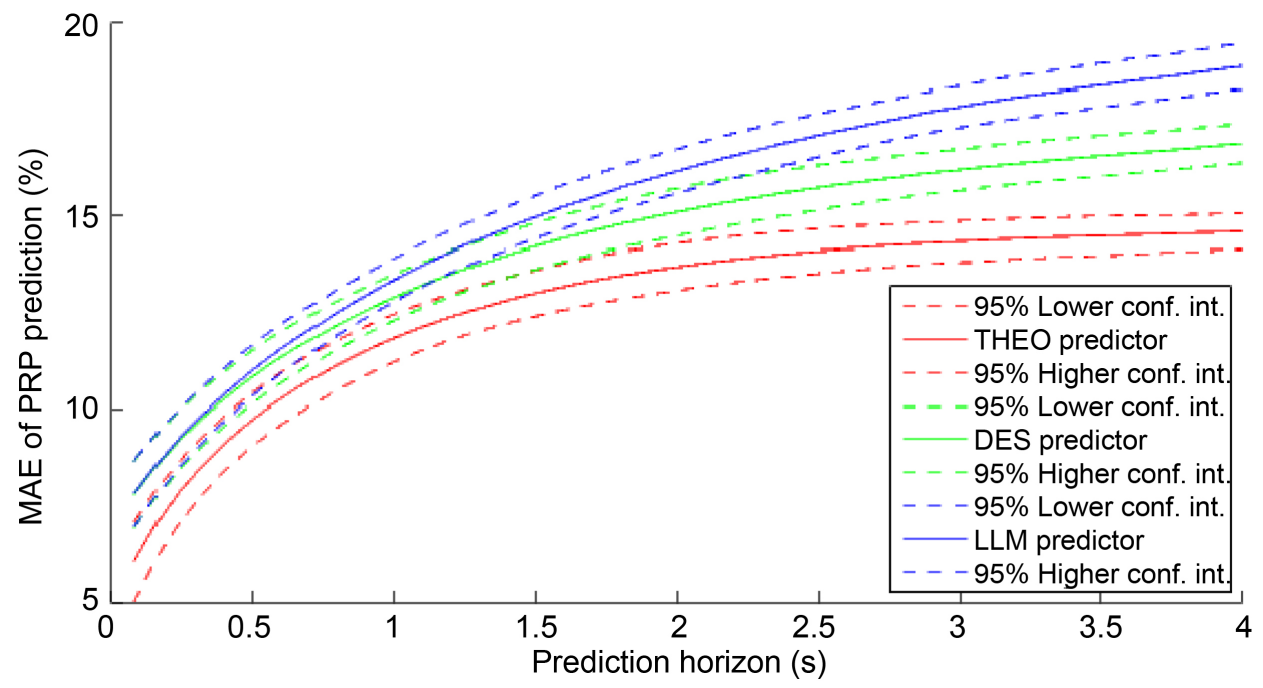

Figure 7. 95\% confidence intervals for PRP prediction errors for 500-byte long packets and the RWP model. 
Table 1. Numerical PRP prediction accuracy for all LQPRs.

\begin{tabular}{ccccc}
\hline Prediction horizon & $1 \mathrm{~s}$ & $2 \mathrm{~s}$ & $3 \mathrm{~s}$ & $4 \mathrm{~s}$ \\
\hline $\begin{array}{c}\text { Mean MAE of PRP prediction } \\
\text { for THEO predictor }\end{array}$ & $12.00 \%$ & $14.07 \%$ & $14.77 \%$ & $15.087 \%$ \\
Mean MAE of PRP prediction & $13.09 \%$ & $15.55 \%$ & $16.71 \%$ & $17.52 \%$ \\
for DES predictor & & & & \\
Mean MAE of PRP prediction & $13.47 \%$ & $16.54 \%$ & $18.21 \%$ & $19.38 \%$ \\
for LLM predictor & & & & \\
Max difference of MAE of PRP prediction & $1.15 \%$ & $1.59 \%$ & $2.11 \%$ & $2.61 \%$ \\
between DES and THEO predictor & & & & \\
Max difference of MAE of PRP prediction & $1.54 \%$ & $2.57 \%$ & $3.65 \%$ & $4.62 \%$ \\
between LLM and THEO predictor & & & & \\
Mean difference of MAE of PRP prediction & $0.39 \%$ & $1.00 \%$ & $1.50 \%$ & $1.89 \%$ \\
between LLM and DES predictor & & & & \\
\hline
\end{tabular}

$1.59 \%$ of the unachievable lower error bound of the THEO predictor for a prediction horizon of $2 \mathrm{~s}$. As expected the DES predictor outperforms the LLM predictor in all scenarios evaluated though there is little difference in accuracy.

There are two noteworthy points to be taken from this paper: large-scale fading is the primary determinant for signal strength prediction and the difference in accuracy between the LLM predictor and the THEO predictor is very small-as evinced by Table 1 above. The LLM, like the DES, can be executed in real-time. We believe that the formulations given in this paper may be applicable to other protocols at other frequencies and environments.

The results given in Table 1 show that real-time accurate LQP is possible for pedestrians in microcellular urban environments within usable prediction horizons. The $95 \%$ confidence intervals are very narrow-typically within the bounds of $\pm 0.6 \%-0.7 \%$ probability.

It was demonstrated that LQP accuracy is somewhat better for longer packets albeit we observed in subsequent trials that this effect becomes less marked.

\section{References}

[1] Schiller, J. (2000) Mobile Communications. Addison-Wesley Professional.

[2] Goncalves, A., Silva, C. and Morreale, P. (2014) Design of a Mobile Ad Hoc Network Communication App for Disaster Recovery. 28th International Conference on Advanced Information Networking and Applications Workshops (WAINA), 13-16 May 2014, 121-126.

[3] Jadhav, S.S., Kulkarni, A.V. and Menon, R. (2014) Mobile Ad-Hoc Network (MANET) for Disaster Management. Eleventh International Conference on Wireless and Optical Communications Networks ( WOCN), 11-13 September 2014, 1-5. http://dx.doi.org/10.1109/WOCN.2014.6923074 
[4] Reijers, N., Cunningham, R., Meier, R., Hughes, B., Gaertner, G. and Cahill, V. (2002) Using Group Communication to Support Mobile Augmented Reality Applications. Presented at Fifth IEEE International Symposium on Object-Oriented Real-Time Distributed Computing, Crystal City.

[5] Goff, T., Abu-Ghazaleh, N.B., Phatak, D.S. and Kahvecioglu, R. (2001) Preemptive Routing in Ad Hoc Networks. International Conference on Mobile Computing and Networking, Rome, 43-52. http://dx.doi.org/10.1145/381677.381682

[6] Jiang, S., He, D. and Rao, J. (2005) A Prediction-Based Link Availability Estimation for Routing Metrics in MANETs. IEEE/ACM Transactions on Networking, 13, 1302-1312. http://dx.doi.org/10.1109/TNET.2005.860094

[7] McDonald, A.B. and Znati, T. (1999) A Path Availability Model for Wireless Ad-Hoc Networks. Presented at IEEE Wireless Communications and Networking Conference, New Orleans.

[8] Lee, S.J., Su, W. and Gerla, M. (1999) Ad Hoc Wireless Multicast with Mobility Prediction. Presented at IEEE International Conference on Computer Communications and Networks, Boston.

[9] Qin, L. and Kunz, T. (2002) Pro-Active Route Maintenance in DSR. ACM SIGMOBILE Mobile Computing and Communications Review, 6, 79-89.

http://dx.doi.org/10.1145/581291.581302

[10] Paul, K., Bandyopadhyay, S., Mukherjee, A. and Saha, D. (1999) Communication-Aware Mobile Hosts in Ad-Hoc Wireless Networks. International Conference on Personal Wireless Communications, Jaipur, 17-19 February 1999, 83-87. http://dx.doi.org/10.1109/icpwc.1999.759590

[11] Gaertner, G., Nuallain, E.O. and Cahill, V. (2008) Extending Wireless Network Simulators to Support Realistic Simulations of 802.11 MANETs. WiCOM, Dalain, 12-14 October 2008, 1-5. http://dx.doi.org/10.1109/wicom.2008.650

[12] Punnoose, R.J., Nikitin, P.V. and Stancil, D.D. (2000) Efficient Simulation of Ricean Fading within a Packet Simulator. Proceedings of the Vehicular Technology Conference, Tokyo, 24-28 September 2000. http://dx.doi.org/10.1109/vetecf.2000.887108

[13] Agarwal, S., Ahuja, A., Singh, J.P. and Shorey, R. (2000) Route-Lifetime Assessment Based Routing (RABR) Protocol for Mobile Ad-Hoc Networks. Presented at IEEE International Conference on Communications, New Orleans, 18-22 June 2000. http://dx.doi.org/10.1109/icc.2000.853783

[14] (1999) ANSI/IEEE Standard 802.11: International Standard ISO/IEC 8802-11: 1999(E), Part 11: Wireless LAN Medium Access Control (MAC) and Physical Layer (PHY) Specifications.

[15] Harvey, A.C. (1993) Time Series Models. 2nd Edition, The MIT Press.

[16] Gudmundson, M. (1991) Correlation Model for Shadow Fading in Mobile Radio Systems. Electronics Letters, 27, 2145-2146. http://dx.doi.org/10.1049/el:19911328

[17] Wang, Z., Tameh, E.K., Nix, A.R. and Gasparini, O. (2004) A Joint Shadowing Process Model for Multihop/Ad-Hoc Networks in Urban Environment. Presented at Wireless World Research Forum, Oslo.

[18] Tepedelenlioglu, C., Abdi, A., Giannakis, G. and Kaveh, M. (2001) Estimation of Doppler Spread and Signal Strength in Mobile Communications with Applications to Handoff and Adaptive Transmission. Wireless Communications and Mobile Computing, 1, 221-242. http://dx.doi.org/10.1002/wcm.1

[19] Nakabayashi, H. and Kozono, S. (1998) Autocorrelation Characteristics for Received Sig- 
nal-Level in a Wide-Band in a Wide-Band Mobile Radio Channel. Transactions IEICE.

[20] Kozono, S. (1994) Received Signal-Level Characteristics in a Wide-Band Mobile Radio Channel. IEEE Transactions on Vehicular Technology, 43, 480-486. http://dx.doi.org/10.1109/25.312798

[21] Goldsmith, A. (2005) Wireless Communications. Cambridge University Press, Cambridge. http://dx.doi.org/10.1017/CBO9780511841224

[22] Stueber, G.L. (2001) Principles of Mobile Communication. 2nd Edition, Kluwer Academic Publishers, Boston.

[23] Gaertner, G. and Nuallain, E.O. (2007) Using the Rice and Nakagami Distributions to Model Wideband Small-Scale Fading in Urban Microcells. IEEE Transactions on Vehicular Technology, 56, 3621-3630. http://dx.doi.org/10.1109/TVT.2007.901857

[24] Goldsmith, A., Greenstein, L. and Foschini, G. (1994) Error Statistics of Real-Time Power Measurements in Cellular Channels with Multipath and Shadowing. IEEE Transactions on Vehicular Technology, 43, 439-446. http://dx.doi.org/10.1109/25.312802

[25] Lee, W.C. (1985) Estimate of Local Average Power of a Mobile Radio Signal. IEEE Transactions on Vehicular Technology, 34, 22-27. http://dx.doi.org/10.1109/T-VT.1985.24030

[26] Kay, S.M. (1993) Fundamentals of Statistical Signal Processing, Volume 1: Estimation Theory. Prentice Hall, New York.

[27] Yates, R.D. and Goodman, D.J. (2005) Probability and Stochastic Processes. 2nd Edition, John Wiles and Sons, Hoboken.

[28] Madisetti, V.K. and Williams, D. (1997) The Digital Signal Processing Handbook. CRC Press, Boca Raton.

[29] Hines, W.W., Montgomery, D.C., Goldsman, D.M. and Borror, C.M. (2002) Probablity and Statistics in Engineering. 4th Edition, John Wiley and Sons, Hoboken.

[30] Bailey, D.H. and Gerguson, H.R.P. (1998) A Strassen-Newton Algorithm for High-Speed Parallelizable Matrix Inversion. ACM/IEEE Conference on Supercomputing, Orlando, 14-18 November 1998, 419-424.

[31] Tepedelenlioglu, C. and Gao, P. (2005) Estimators of the Nakagami-M Parameter and Performance Analysis. IEEE Transactions on Wireless Communications, 4, 519-527. http://dx.doi.org/10.1109/TWC.2004.843017

[32] Gaertner, G., Nuallain, E.O., Butterly, A., Singh, K. and Cahill, V. (2004) 802.11 Link Quality and Its Prediction-An Experimental Study. In: Niemegeers, I. and de Groot, S.H., Eds., Personal Wireless Communications, Lecture Notes in Computer Science, Springer, Berlin, 147-163. http://dx.doi.org/10.1007/978-3-540-30199-8_13

[33] Berg, J.E., Bownds, R. and Lotse, F. (1992) Path Loss and Fading Models for Microcells at 900 MHz. IEEE Vehicular Technology Conference, Denver, 10-13 May 1992, 666-671. http://dx.doi.org/10.1109/vetec.1992.245484

[34] PalChaudhuri, S., Boudec, J.Y.L. and Vojnovic, M. (2005) Perfect Simulations for Random Trip Mobility Models. Annual Simulation Symposium, San Diego, 4-6 April 2005, 72-79. http://dx.doi.org/10.1109/anss.2005.33

[35] Saha, A.K. and Johnson, D.B. (2004) Modeling Mobility for Vehicular Ad Hoc Networks. Workshop on Vehicular Ad Hoc Networks (VANET), Philadelphia, 26 September-1 October 2004, 91-92. http://dx.doi.org/10.1145/1023875.1023892

[36] Administration, F.H. (2003) Manual on Uniform Traffic Control Devices (MUTCD). US DOTransportation.

[37] Wakim, C., Capperon, S. and Oksman, J. (2004) A Markovian Model of Pedestrian Beha- 
vior. IEEE International Conference on Systems, Man and Cybernetics, 4, 4028-4033.

[38] Kurkowski, S., Camp, T. and Colagrosso, M. (2005) MANET Simulation Studies: The Incredibles. ACM SIGMOBILE Mobile Computing and Communications Review, 9, 50-61. http://dx.doi.org/10.1145/1096166.1096174

Submit or recommend next manuscript to SCIRP and we will provide best service for you:

Accepting pre-submission inquiries through Email, Facebook, LinkedIn, Twitter, etc. A wide selection of journals (inclusive of 9 subjects, more than 200 journals)

Providing 24-hour high-quality service

User-friendly online submission system

Fair and swift peer-review system

Efficient typesetting and proofreading procedure

Display of the result of downloads and visits, as well as the number of cited articles

Maximum dissemination of your research work

Submit your manuscript at: http://papersubmission.scirp.org/

Or contact jcc@scirp.org 